\title{
Mapping genetic determinants of host susceptibility to Pseudomonas aeruginosa lung infection in mice
}

\author{
Maura De Simone ${ }^{1 \dagger}$, Lorenza Spagnuolo ${ }^{1 \dagger}$, Nicola Ivan Lorè ${ }^{1}$, Cristina Cigana ${ }^{1}$, Ida De Fino ${ }^{1}$, Karl W. Broman², \\ Fuad A. Iraqi ${ }^{3+}$ and Alessandra Bragonzi ${ }^{1 *+}$
}

\begin{abstract}
Background: $P$. aeruginosa is one of the top three causes of opportunistic human bacterial infections. The remarkable variability in the clinical outcomes of this infection is thought to be associated with genetic predisposition. However, the genes underlying host susceptibility to $P$. aeruginosa infection are still largely unknown.

Results: As a step towards mapping these genes, we applied a genome wide linkage analysis approach to a mouse model. A large F2 intercross population, obtained by mating $P$. aeruginosa-resistant $\mathrm{C} 3 \mathrm{H} / \mathrm{HeO}$ J, and susceptible $\mathrm{A} / \mathrm{J}$ mice, was used for quantitative trait locus (QTL) mapping. The F2 progenies were challenged with a $P$. aeruginosa clinical strain and monitored for the survival time up to 7 days post-infection, as a disease phenotype associated trait. Selected phenotypic extremes of the F2 distribution were genotyped with high-density single nucleotide polymorphic (SNP) markers, and subsequently QTL analysis was performed. A significant locus was mapped on chromosome 6 and was named P. aeruginosa infection resistance locus 1 (Pair/1). The most promising candidate genes, including Dok1, Tacr1, Cd207, Clec4f, Gp9, Gata2, Foxp1, are related to pathogen sensing, neutrophils and macrophages recruitment and inflammatory processes.
\end{abstract}

Conclusions: We propose a set of genes involved in the pathogenesis of $P$. aeruginosa infection that may be explored to complement human studies.

Keywords: P. aeruginosa, Pneumonia, Linkage analysis, Murine model, Host susceptibility, QTL mapping, Candidate genes

\section{Background}

P. aeruginosa is a versatile Gram-negative bacterium mainly implicated in pneumonia in intensive care settings and in patients with neutropenia, advanced AIDS, bronchiectasis, hereditary cystic fibrosis (CF) disease and advanced chronic obstructive pulmonary disease (COPD). Hospital-acquired pneumonia (HAP) occurs predominantly in patients with intubation and mechanical ventilation in an intensive care unit (ICU) $[1,2]$. The risk of fatality in these patients is generally high but variable even

\footnotetext{
* Correspondence: bragonzi.alessandra@hsr.it

Maura De Simone and Lorenza Spagnuolo: These authors have equal contributions and share first authorship.

Fuad A. Iraqi and Alessandra Bragonzi: These authors have equal contributions and share senior authorship.

${ }^{\dagger}$ Equal contributors

'Infection and Cystic Fibrosis Unit, IRCCS San Raffaele Scientific Institute, Milan, Italy

Full list of author information is available at the end of the article
}

with the correct use of antibiotics. Similarly to other infectious agents, exposure to $P$. aeruginosa results in a range of mild to severe clinical symptoms which can lead to fatal bacteremia in critically ill patients [3].

In general, the pathophysiology of human infections is a complex and dynamic process that depends on several factors: the genetic predisposition, the co-morbid conditions, the host immune response, the type of pathogen, and the site and extent of infection [4]. Most of the studies in the field of $P$. aeruginosa have been concentrated on the type of strains and the virulence factors [5-10]. A major contributing cause of $P$. aeruginosa predominance and pathogenesis was attributed to its ability to adapt to many different environments and cause a wide range of diseases in humans [11]. Less attention has been paid to host genetic predisposition as a contributing factor to the clinical outcome of $P$. 
aeruginosa infection. However, evidence of genetic predisposition is emerging in the CF and COPD populations. In CF, it has been shown that the progression and severity of pulmonary disease do not appear to correlate exclusively with specific mutations in the causative gene cystic fibrosis transmembrane conductance regulator (CFTR) but appear to be largely dependent on other modifier genes $[12,13]$. In COPD, several host candidate genes have been proposed as causing the acceleration of lung function decline but have never been associated with $P$. aeruginosa infection $[14,15]$. Regarding other patients at risk of $P$. aeruginosa infection, no study has yet been reported. The size of cohorts, strong but often unknown environmental influences, poor diagnosis, and lack of repeatability limit scientific advancements in understanding the genetic basis of susceptibility to $P$. aeruginosa diseases in humans.

Inbred mice, with defined and reproducible genetics, can be maintained in standardized environments and exposed to identical challenges, unlike human populations. These aspects allow us to directly correlate the phenotypic response to the specific genotype. Inbred mice have been successfully adopted to allow us to understand the genetic basis of differential susceptibility to infections (e.g. bacteria, viruses and parasites) $[16,17]$. When genes are identified in a mouse model, human orthologue genes can subsequently be identified. In the context of $P$. aeruginosa infection, variability in survival, magnitude of inflammatory response, bacterial clearance and lung damage have been reported in various inbred strains [18-22]. Recently, we also showed that the genetic background of the host influences the response to $P$. aeruginosa infection [23]. These data were obtained by ranking nine inbred murine strains characterized by a wealth of genetic and phenotypic diversity. In particular, $\mathrm{A} / \mathrm{J}$ and $\mathrm{C} 3 \mathrm{H} / \mathrm{HeOuJ}$ showed the most deviant clinical (e.g. body weight and mortality) and immunological phenotypes (e.g. cytokines and innate immune cells response). These results provided a basis for mapping genomic regions underlying host susceptibility to $P$. aeruginosa infection. Among different traits recorded, mortality caused by bacteremia was considered as the ultimate clinical expression of the deleterious clash between the host immune response and invasive microorganisms. Consequently, in this study we generated an informative F2 population by crossing between the parental strains, including resistant $\mathrm{C} 3 \mathrm{H} / \mathrm{HeOuJ}$ and susceptible $\mathrm{A} / \mathrm{J}$ mice and subsequently performed quantitative trait loci (QTLs) mapping analysis. We identified a genetic locus on murine chromosome 6 , designated $P$. aeruginosa infection resistance locus 1 (Pairl1), that has major effect on the susceptibility to $P$. aeruginosa infection and risk of bacteremia.

\section{Methods}

\section{Ethic statement}

Animal studies were conducted according to protocols approved by San Raffaele Scientific Institute (Milan, Italy) Institutional Animal Care and Use Committee (IACUC) and adhered strictly to the Italian Ministry of Health guidelines for the use and care of experimental animals (Permit number: 502). Research on the bacterial isolates AA2, from the individual with $\mathrm{CF}$, used for animal experiments, has been approved by the Ethics Commission of Hannover Medical School, Germany. The patients and parents gave informed verbal consent before the sample collection. Approval for storing of biological materials was obtained by the Ethics Commission of Hannover Medical School, Germany.

\section{Bacterial strain}

$P$. aeruginosa clinical isolate AA2 was described before $[6,23,24]$. The strain was cultured in trypticase soy broth (TSB) and plated on trypticase soy agar (TSA).

\section{Mice, breeding and $P$. aeruginosa lung infection}

Based on our previous study, we have determined that $\mathrm{A} / \mathrm{J}$ and $\mathrm{C} 3 \mathrm{H} / \mathrm{HeOuJ}$ are susceptible and resistant to $P$. aeruginosa infection [23], respectively $7-8$ weeks old male and female mice of $\mathrm{A} / \mathrm{J}$ and $\mathrm{C} 3 \mathrm{H} / \mathrm{HeOuJ}$ were purchased from Jackson Laboratory, and used as the parental founders of F2 mapping population. An outcross between the two founders was conducted and subsequently the members of the first generation (F1 population) were intercrossed to generate $400(\mathrm{~A} / \mathrm{J} \mathrm{X} \mathrm{C3H/}$ $\mathrm{HeOuJ}) \mathrm{F} 2$ mice. $\mathrm{A} / \mathrm{J},(n=22) \mathrm{C} 3 \mathrm{H} / \mathrm{HeOuJ}(n=26)$, and $(\mathrm{A} / \mathrm{J} \times \mathrm{C} 3 \mathrm{H} / \mathrm{HeOuJ}) \mathrm{F} 2$ mice $(n=400)$ were intratracheal injected with a dose of $5 \times 10^{6}$ of the planktonic $P$. aeruginosa clinical isolate AA2, and monitored for survival up to 7 days according with established procedures [23].

Mice were maintained in pathogen free conditions during breeding and transferred in biosafety level 3 stabulary for infection experiments. Mice were observed daily for clinical signs including coat quality, posture, ambulation, and hydration status.

Statistical significance by Mantel-Cox test was performed to compare the survival between pairs. The data are pooled at least from three independent experiments.

\section{Genotyping}

We applied a selective genotyping approach, as suggested by Darvasi and Soller [25], whose power was successfully proven and confirmed in several previous studies [26-30]. We genotyped only the phenotypic extremes of the entire F2 outcross population. A total of 160 mice of the F2 population (20\% of highly and $20 \%$ lowly susceptible) were genotyped and genotypes were randomized by meiosis. 
High molecular genomic DNA samples of the 160 F2 mice and the parental mouse strains were extracted from tail biopsies with the DNeasy Blood and Tissue (Qiagen, Hilden, Germany). Samples were analyzed for purity $\left(\mathrm{A}_{260} / \mathrm{A}_{280}\right)$ and DNA content was evaluated by NanoDrop measurement. The genomic DNA samples of the F2 progeny were genotyped with MegaMUGA mouse array which consists 77,000 SNP markers based on the Illumina Infinium platform [31]. The SNP genotype was conducted at Neogen company (Neogen/Geneseek, Lincoln, NE, USA). The SNP markers are distributed across the entire genome with an average spacing of $33 \mathrm{~Kb}$.

\section{QTL analysis}

To map QTL contributing to survival, we used HaleyKnott regression [32], including sex as an additive covariate (that is, allowing for a shift in average survival between the two sexes, but assuming that the effect of a QTL is the same in both sexes). Statistical significance was assessed by a permutation test [33], correcting for the multiple statistical tests inherent in the scan of the genome. Sample size was chosen to give $80 \%$ power to detect a QTL explaining $~ 5 \%$ of the phenotypic variance. A confidence interval for the location of the inferred QTL was derived as a 1.5-LOD support interval [34]. Calculations were performed with R/qtl [35], an add-on package to the general statistical software $R$ ( $R$ Development Core Team 2015).

\section{Candidate genes analysis}

The public database Mouse Genome Informatics (MGI, http://www.informatics.jax.org/) was used for the identification of suggested genes within the QTL interval and for prioritization together with Endeavour [36, 37]. To generate the training sets of genes employed for the analysis, we used the 'key word' approach described previously [38, 39].

\section{Statistical analysis}

We used Mantel-Cox test to compare the survival between pairs. Tests were considered statistically significant when the significance level was $\leq 0.05$.

\section{Results}

\section{Susceptibility to $P$. aeruginosa pulmonary infection in (A/JxC3H/HeOuJ) F2 progenies}

Our experiments established that the intratracheal injection of $5 \times 10^{6} \mathrm{CFU}$ of the $P$. aeruginosa clinical isolate AA2 causes the death of A/J mice within $24 \mathrm{~h}$, whereas a set of $\mathrm{C} 3 \mathrm{H} / \mathrm{HeOuJ}$ mice $(\sim 40 \%)$ survives for more than 7 days (Fig. 1). The selected A/J and $\mathrm{C} 3 \mathrm{H} / \mathrm{HeOuJ}$ parental inbred mouse strains were highly and significantly divergent in the risk of sepsis $(p<0.0001$, MantelCox test). To analyse the potential contribution of genetic factors to $P$. aeruginosa infection, the $\mathrm{F} 1$ population and subsequently $400(\mathrm{~A} / \mathrm{JxC} 3 \mathrm{H} / \mathrm{HeOuJ}) \mathrm{F} 2$ mice were generated by reciprocal mating of susceptible $\mathrm{A} / \mathrm{J}$ and resistant $\mathrm{C} 3 \mathrm{H} / \mathrm{HeOuJ}$ mice. The entire $\mathrm{F} 2$ population was infected with $P$. aeruginosa as described above and survival of each mouse was recorded over a period of 7 days postinfection. F2 progenies showed a survival which was significantly higher than the susceptible strain A/J, but lower when compared to $\mathrm{C} 3 \mathrm{H} / \mathrm{HeOuJ}$ parental lines (Fig. 1). Then, to collect data for the subsequent genome analysis, the F2 population was sorted based on the phenotypic extremes - the most susceptible (F2-S) (20\% of F2 population $n=80$ ) and most resistant (F2-R) (20\% of F2 population $n=80)$-. The selected F2-S showed a significantly lower survival compared to $\mathrm{C} 3 \mathrm{H} / \mathrm{HeOuJ}$ mice (Hazard Ratio 0.03057, 95 \% CI 0.01386 to 0.06741 ; $p<0.0001$, Mantel-Cox test) but similar to A/J mice (Hazard Ratio 0.4345 , 95 \% CI 0.1288 to 1.466) while F2-R showed a significantly higher survival compared to the parental $\mathrm{C} 3 \mathrm{H} / \mathrm{HeOuJ}$ (Hazard Ratio 2.969, $95 \%$ CI 1.382 to $6.378 ; p=0.0053$, Mantel-Cox test) and A/J mice (Hazard Ratio 688.9, 95 \% CI210.3 to 2256; $p<0.0001$,Mantel-Cox test). The rest of the F2 population (Fig. 1 as not detected genotype, F2-nd) showed survival kinetics that were significantly different from F2-S and F2 -R (Hazard ratio 0.004038, $95 \%$ CI 0.002248 to 0.007256 for F2-S, and Hazard ratio $18.56,95 \%$ CI 12.65 to 27.21 for F2-R; $p<0.0001$, MantelCox test) and the parental lines (Hazard Ratio 0.3129, $95 \%$ CI 0.1486 to 0.6589 for $\mathrm{C} 3 \mathrm{H} / \mathrm{HeOuJ} ; p=0.0022$ Mantel-Cox test, and Hazard Ratio 5785, 95 \% CI 1549 to 21606 for A/J; $p<0.0001$, Mantel-Cox test). Finally the entire F2 population $(n=400)$ showed a significantly higher survival compared to the parental A/J (Hazard Ratio

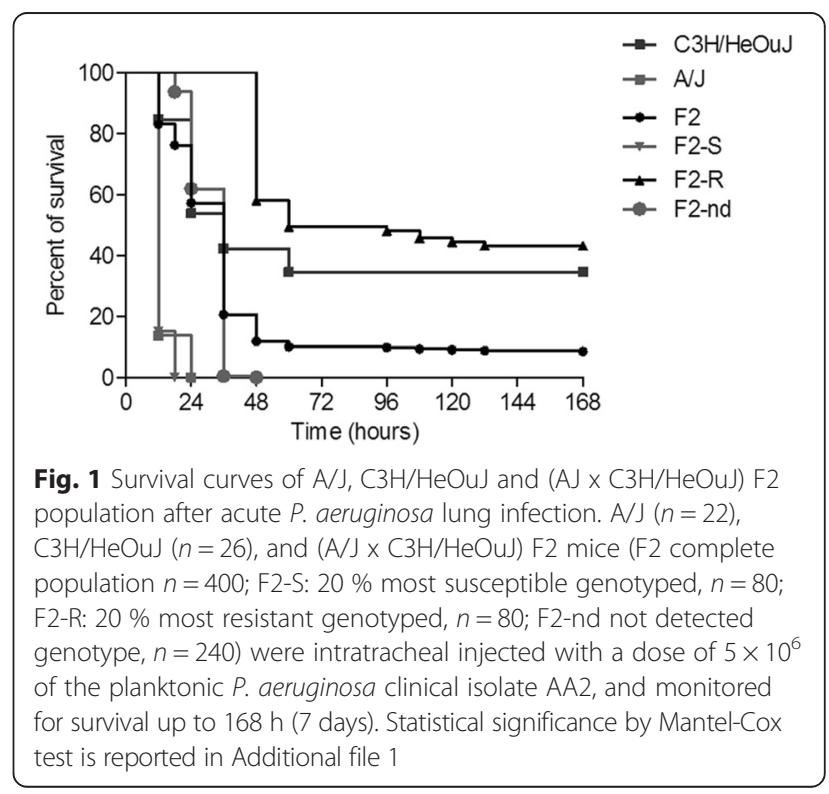


49.79, 95 \% CI 19.16 to $129.4 ; p<0.0001$, Mantel- Cox test) a slightly significantly lower survival when compared to the parental C3H/HeOuJ (Hazard Ratio 0.5007, 95 \% CI 0.3270 to $0.8615 ; p=0.0104$ Mantel- Cox test). The complete statistical analysis is reported in Additional file 1 (Table S1). The distribution histogram of the survival time in the F2 population, with values ranging from 0,5 to 7 days, is shown in Fig. 2b, along with the distribution in $\mathrm{A} / \mathrm{J}$ mice (Fig. 2a) and $\mathrm{C} 3 \mathrm{H} / \mathrm{HeOuJ}$ mice (Fig. 2c).

Additional analysis showed that high numbers of CFUs (average of $1 \times 10^{6}$ in the whole spleen) were present in the moribund mice, both parental and their progenies, indicating sepsis as the cause of death (data not shown).

\section{Mapping the genetic determinants to $P$. aeruginosa susceptibility}

In the next phase, we proceeded to map the genetic factors that contributed to susceptibility to the $P$. aeruginosa acute lung infection. The survival time was used as

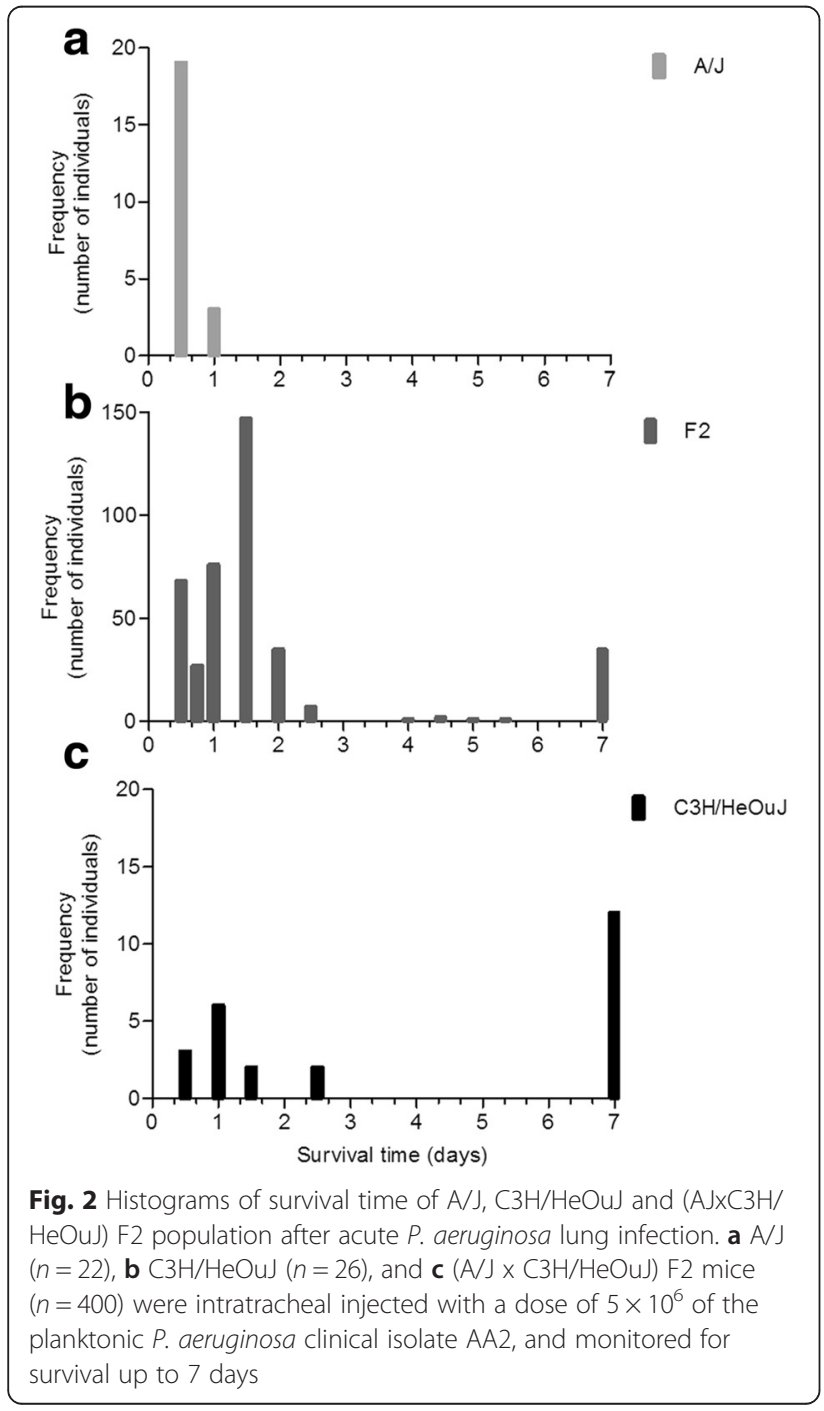

the main phenotypic trait for mapping QTL associated with host susceptibility to this opportunistic pathogen. In order to maximize the ability to detect the QTLs, we took advantage of the selective genotyping approach, described previously [25] and successfully applied in several studies [27-29]. The phenotypic extremes F2-S and F2-R were genotyped with about $10 \mathrm{~K}$ informative SNP markers selected from MegaMuga SNP array, and subsequently QTL mapping was performed. We identified a significant QTL on chromosome $6(p=0.037)$ at map position 90.8 Mbp with LOD Score $=4.3$, and with a 1.5-LOD support genomic interval of 81.5-102.2 Mbp (Fig. 3). The SNPs markers used to genotype the locus of interest on chromosome 6 are reported in Additional file 2 (Table S2). We defined the mapped QTL on chromosome 6 as Pairl1 for P. aeruginosa infection resistance locus 1 .

The effect of the locus on survival is presented in Additional file 3 (Figure S1). The locus shows some degree of over-dominance, with the heterozygotes surviving longer than the homozygotes (A/J homozygotes versus heterozygotes $p=0.0056$ Mantel-Cox test; C3H/ $\mathrm{HeOuJ}$ homozygotes versus heterozygotes $p<0.0001$, Mantel-Cox test) and a transgressive allele segregation with the $\mathrm{A} / \mathrm{J}$ homozygotes surviving longer than the $\mathrm{C} 3 \mathrm{H} /$ $\mathrm{HeOuJ}$ homozygotes. This is the opposite what would be expected from the parental strains' phenotypes.

Identification and functional classification of candidate genes A total of 323 genes within the Pairl1 interval were identified from the public database (http://informatics.jax.org/), as described in Material and methods and classified as protein coding genes $(n=198)$, RNA genes $(n=50)$, unclassified genes $(n=45)$ and unclassified non-coding RNA genes $(n=30)$. The full list of genes in the interval is reported in Additional file 4 (Table S3). Gene prioritization was: i) carried out by integrative computational analysis of public and private genomic data [38], ii) based on training lists of genes involved in relevant biological and pathological processes and iii) ranked based on literature research.

To prioritize our genes, we used Endeavour and the MGI database (http://informatics.jax.org/). For Endeavour, candidates genes are first ranked according to their similarity to a list of known disease training genes involved in lung infection, bacteremia, pneumonia, sepsis, chemotaxis, $P$. aeruginosa, innate immune response, pattern recognition receptor, neutrophils, complement, $\mathrm{CF}$, modifier genes in CF, and COPD (Additional file 5A: Table S4A). For the MGI database, we based the search on different keywords (Lung, Infection, Respiratory AND Infection) and gene ontology terms (Immune AND Response, Cytokines, Chemotaxis) (Additional file 5B: Table S4B). Interestingly, the two different approaches 


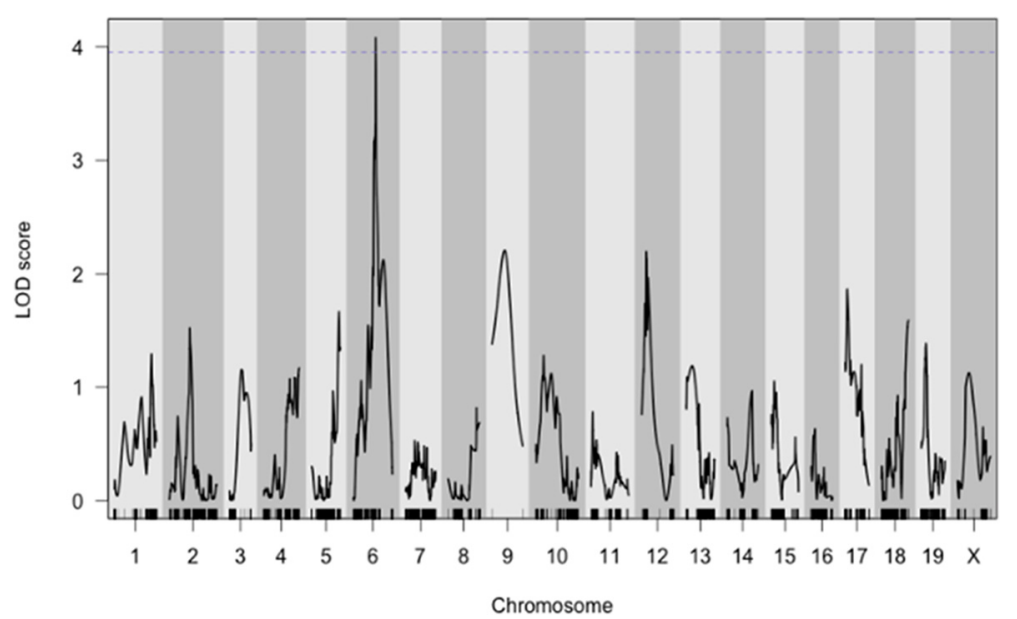

Fig. 3 QTL scan showing LOD score (Y-axis) and genome position (X-axis) for susceptibility to $P$. aeruginosa infection. The $x$ axis shows chromosome numbers, the $y$ axis shows the statistical significance of the association measured as LOD, logarithm of the odds favouring linkage, a score that measures the strength of evidence for the presence of a QTL among the 160 mice of the F2 population, including F2-S and F2-R. The horizontal line is plotted at the $5 \%$ significance threshold. One significant QTL ( $p=0.037$ ) named Pairl 1 was mapped on chromosome 6 at 90.8 Mbp with 4.3 LOD score, with a 1.5-LOD drop support interval of 81.5-102.2 Mbp

revealed several redundant genes and consequently, we proceeded with a systematic literature research to confirm a possible role in susceptibility to $P$. aeruginosa infection. The candidate genes in Table 1 are those revealed by the literature research as having a putative role in host defense to infection. In particular, the most promising candidate genes (Dok1, Tacr1, Cd207, Clec4f, Gp9, Gata2, Foxp1) were those involved in pathogen sensing, neutrophils and macrophages recruitment, and inflammatory process. Our prioritization of results depends on the adopted criteria for the bioinformatics analysis and on the bioinformatics tool

Table 1 The list of the most promising candidate genes identified in the Pairl1 locus

\begin{tabular}{|c|c|c|}
\hline Gene name & Biological function & References \\
\hline Dok1 & $\begin{array}{l}\text { Adaptor protein involved in: } \\
\text { - lung homeostasis } \\
\text { - negative regulation of LPS signaling } \\
\text { - Downregulation of its expression in in vitro } \\
\text { models }\end{array}$ & {$[28,29]$} \\
\hline Tacrl & $\begin{array}{l}\text { Receptor for neuropeptide tachykinin involved in: } \\
\text { - Cytokines and chemokines expression } \\
\text { - Chemotaxis and neutrophils activation } \\
\text { - P. aeruginosa corneal infection }\end{array}$ & [30-32] \\
\hline $\begin{array}{l}\text { Cd207 } \\
\text { Clecf4 }\end{array}$ & $\begin{array}{l}\text { Members of C-type lectin superfamily involved in: } \\
\text { - Pathogen sensing of virus, fungi and bacteria }\end{array}$ & {$[33-35]$} \\
\hline Gp9 & $\begin{array}{l}\text { Glycoprotein 9-Glycoprotein lb complex } \\
\text { involved in: } \\
\text { - Leukocytes migration } \\
\text { - Inflammation } \\
\text { - Neutrophils extracellular trap formation }\end{array}$ & {$[36]$} \\
\hline $\begin{array}{l}\text { Foxp1 } \\
\text { Gata2 }\end{array}$ & $\begin{array}{l}\text { Transcriptional factors involved in: } \\
\text { - Cytokines expression }\end{array}$ & {$[37,38]$} \\
\hline
\end{tabular}

itself. Therefore, it cannot be excluded that other possible candidate genes present in our list could somehow play a role in $P$. aeruginosa infection.

\section{Discussion}

Phenotypic variations of host response to $P$. aeruginosa infection have been demonstrated in several inbred mouse strains [18-22] and in the most recent Collaborative Cross population [40 ]. In our earlier work, we showed that classical inbred strains of mice manifest an extreme response - highly resistant or highly susceptible - to $P$. aeruginosa infection [23]. In particular, the risk of fatality in A/J mice was high after exposure to $P$. aeruginosa while $\mathrm{C} 3 \mathrm{H} / \mathrm{HeOuJ}$ showed milder clinical symptoms associated to higher resolution of the infection. However, the genetic component of this trait is still poorly understood. For the first time to our knowledge, in this study, we localized a genetic determinant of susceptibility to $P$. aeruginosa respiratory infection. First, we generated an informative F2 population by crossing the resistant $\mathrm{C} 3 \mathrm{H} / \mathrm{HeOuJ}$ and susceptible A/J mice. Next, we scored this F2 population for survival time, as the main associated disease phenotypic trait, and subsequently used it for QTL mapping. The $P$. aeruginosa dose for infection of the newly generated populations was selected according to the most deviant differences in survival between parental lines. The F2 population showed a wide range of responses in terms of survival compared to the parental lines. For this study, we used $P$. aeruginosa isolate sampled at the onset of infection from a CF patient and fully characterized in previous studies [41-44]. Although the P. aeruginosa strain 
was selected on the basis of its similarity to environmental strains, including genotypic and phenotypic features, it is possible that this particular strain may affect several physiological parameters and subsequent analysis.

Our genetic linkage approach was successful in identifying for the first time the Pairl1 locus, on murine chromosome 6 , linked to susceptibility to $P$. aeruginosa pulmonary infection with a genome wide significance of $95 \%(p<0.05)$. Here, the gene prioritization approach was used to rank genes involved in the susceptibility to $P$. aeruginosa infection. This approach was applied successfully in candidate gene analysis in other diseases such as obesity, Type 2 diabetes, and asthma [45, 46$]$. The most promising candidate genes included in the Pairl1 locus, namely Dok1, Tacr1, Cd207, Clec4f, Gp9, Gata2 and Foxp1, are mainly involved in pathogen sensing, neutrophils recruitment and cytokines response.

In more detail, as Dok1 (Docking protein1) belongs to a family of adaptor proteins, it plays a critical antiinflammatory role in lung homeostasis. It is involved in negative regulation of LPS signaling and its expression is downregulated after $P$. aeruginosa infection in vitro [47, 48]. Together with Dok 2 and Dok 3, it plays essential roles in negative regulation of a wide variety of signaling pathways in both innate and adaptive immunities [49]. Mice knock-out for Dok1 were hypersensitive to LPS showing high levels of TNF $\alpha$ and NO [48] while triple KO mice (Dok1-2-3) showed spontaneous pulmonary inflammation, with hallmarks of asthma, including eosinophilia, goblet cell hyperplasia, and subepithelial fibrosis [50]. Most importantly, downregulation of Dok1 gene expression in human alveolar macrophages after $P$. aeruginosa infection in vitro, indicates its direct involvement in this bacterial infection [48].

The gene Tacr1 (Tachykinin receptor 1) encodes the receptor for the neuropeptide tachykinin and is implicated in mediating a variety of proinflammatory processes, including upregulation of cytokine and chemokine expression, chemotaxis and activation of inflammatory cells, all pathways that seem to be altered in susceptible A/J mice during acute $P$. aeruginosa infection [51]. Moreover, the binding of this receptor with its ligand has been shown to increase the neutrophils adhesion to bronchial epithelial cells [52]. More interestingly, Tacr1 has been implicated in several bacterial infections and in sepsis [53, 54], including $P$. aeruginosa corneal infection [55].

Cd207 (C-type lectin domain family 4 member $\mathrm{K}$ ) gene encodes a type II transmembrane, $\mathrm{C}$-type lectin receptor displaying mannose-binding specificity. It has high affinity for mannose structures abundantly expressed by pathogens, especially by viruses (HIV-1 and HSV) as well as the mycobacterial cell wall component mannosylated lipoarabinomannan (ManLAM) and pathogenic funguses such as Candida species, Malassezia furfur and Saccharomyces cerevisiae [56]. The gene Clec4f shows high similarity with Cd207, and therefore might be involved in the same processes. The importance of these genes in the context of $P$. aeruginosa infection might be seen in the functional association of this C-type lectin receptor with the Mannose Binding Lectin 2 (MBL2) gene, which codes for the MBL, a well-known modifier gene in CF. In fact, polymorphisms in MBL2 are associated with differing level of MBL production, and deficiencies in MBL production have been linked to an increased incidence of infection, [57]. In particular it predisposes to early infection with $P$. aeruginosa which in turn, leads to more severe lung disease [58].

Interestingly, other candidates in the Pairl1 locus play important roles in leukocytes recruitment. Specifically, Gp9 (glycoprotein 9), expressed by platelets, is involved in leukocyte migration, inflammation and promotion of neutrophil extracellular trap formation [59]. In a mouse model of sepsis this glycoprotein complex contributes to a platelet/neutrophil and platelet/monocyte axis with significant consequences to the innate immune response in terms of elevated serum cytokine levels and Mac1 increased expression by neutrophils for their extracellular cell migration. Among the candidate genes, we also identified the transcription factors Foxp1 (Forkhead box P1) and Gata2 (GATA binding protein 2) that are involved in pro-inflammatory cytokines expression, such as IL1ß, IL-6 and IL-17RA correlated to the Th17 pathway (http://www.innatedb.com/) [60].

\section{Conclusions}

We suggest promising candidates for the genetic basis of host susceptibility to $P$. aeruginosa infection. In addition to a better understanding of host pathogen interactions, the characterization of these genes may have significant implications for the discovery of novel possible therapeutic targets and/or prognostic biomarkers complementing human studies.

\section{Ethics}

Animal studies were conducted according to protocols approved by San Raffaele Scientific Institute (Milan, Italy) Institutional Animal Care and Use Committee (IACUC) and adhered strictly to the Italian Ministry of Health guidelines for the use and care of experimental animals (Permit number: 502). Research on the bacterial isolates AA2, from the individual with $\mathrm{CF}$, used for animal experiments, has been approved by the Ethics Commission of Hannover Medical School, Germany. The patients and parents gave informed verbal consent before the sample collection. Approval for storing of biological materials was obtained by the Ethics Commission of Hannover Medical School, Germany. 


\section{Consent to publish}

Not applicable.

\section{Availability of data and materials}

The data sets supporting the results of this article are included within the article and its additional files.

\section{Additional files}

Additional file 1: Statistical comparison of survival time between A/J, $\mathrm{C} 3 \mathrm{H} / \mathrm{HeOuJ}$ and (AJxC3H/HeOuJ) F2 population after acute $P$. aeruginosa lung infection. (DOCX $15 \mathrm{~kb}$ )

Additional file 2: Recombination map position of 38 SNPs markers in the locus of interest of F2 Chromosome 6. (DOCX $16 \mathrm{~kb}$ )

Additional file 3: Effect of the Pair/1 locus on survival in $\mathrm{A} / \mathrm{J}, \mathrm{C} 3 \mathrm{H} /$ HeOuJ and heterozygotes. (DOCX 106 kb)

Additional file 4: List of all the genes within the Pairl1 locus obtained from public database Mouse Genome Informatics (MGl, http://www.informatic.jax.org). (DOCX 66 kb)

Additional file 5: List of the most promising candidates genes for susceptibility to acute $P$. aeruginosa pulmonary infection. (DOCX $27 \mathrm{~kb}$ )

\section{Abbreviations}

CF: cystic fibrosis; CFTR: cystic fibrosis transmembrane conductance regulator; COPD: chronic obstructive pulmonary disease; Cd207 and Clecf4: C-type lectin domain family 4 member $f$ and $K$; Dok1: Docking protein 1: Foxp1: Forkhead box P1: Gata2: GATA binding protein 2: Gp9: glycoprotein 9; ICU: intensive care unit; QTL: quantitative trait locus; Tacr1: Tachykinin receptor 1 .

\section{Competing interests}

The authors declare that they have no competing interests.

\section{Authors' contributions}

MDS, LS, NIL, CC, IDF, KWB performed research; MDS, LS, KWB, FI, AB designed research; MDS, LS, NIL, CC, IDF, KWB, FI, AB analyzed data; MDS, LS, Fl, AB wrote the paper. All authors have read and approved the final version of the manuscript.

\section{Acknowledgments}

The authors thank Prof. B. Tu"mmler (Klinische Forschergruppe, Medizinische Hochschule Hannover, Germany) for supplying the P. aeruginosa AA2 and Dr. Davide Cittaro and Dr. Dejan Lazaveric (Bioinformatics Core Center for Translational Genomics and Bioinformatics, San Raffaele Scientific Institute, Milan, Italy) for their support relative to the genes prioritization approach.

\section{Funding}

This study was supported by Ministero della Salute (project GR/2009/ 1579812) and Italian Cystic Fibrosis Research Foundation (FFC 9/2014) with the contribution of Delegazione FFC di Milano.

\section{Author details}

${ }^{1}$ Infection and Cystic Fibrosis Unit, IRCCS San Raffaele Scientific Institute, Milan, Italy. ${ }^{2}$ Department of Biostatistics \& Medical Informatics, University of Wisconsin, Madison, WI, USA. ${ }^{3}$ Department of Clinical Microbiology and Immunology, Sackler Faculty of Medicine, Tel Aviv University, Ramat Aviv, 69978 Tel Aviv, Israel.

Received: 20 November 2015 Accepted: 28 April 2016 Published online: 11 May 2016

\section{References}

1. Gellatly SL, Hancock RE. Pseudomonas aeruginosa: new insights into pathogenesis and host defenses. Pathog Dis. 2013;67(3):159-73.

2. Lyczak JB, Cannon CL, Pier GB. Establishment of Pseudomonas aeruginosa infection: lessons from a versatile opportunist. Microbes Infect. 2000;2:1051-60.

3. Sligl WI, Dragan T, Smith SW. Nosocomial gram-negative bacteremia in intensive care: epidemiology, antimicrobial susceptibilities and outcomes. Int J Infect Dis. 2015;37:129-134.

4. Cooke GS, Hill AV. Genetics of susceptibility to human infectious disease. Nat Rev Genet. 2001;2(12):967-77.

5. Nguyen D, Singh PK. Evolving stealth: genetic adaptation of Pseudomonas aeruginosa during cystic fibrosis infections. Proc Natl Acad Sci U S A. 2006;103(22):8305-6.

6. Bragonzi A, Paroni M, Nonis A, Cramer N, Montanari S, Rejman J, Di Serio C, Doring G, Tummler B. Pseudomonas aeruginosa microevolution during cystic fibrosis lung infection establishes clones with adapted virulence. Am J Respir Crit Care Med. 2009:180(2):138-45.

7. Cigana C, Curcuru L, Leone MR, lerano T, Lore NI, Bianconi I, Silipo A, Cozzolino F, Lanzetta R, Molinaro A, et al. Pseudomonas aeruginosa exploits lipid $A$ and muropeptides modification as a strategy to lower innate immunity during cystic fibrosis lung infection. PLoS One. 2009:4(12), e8439.

8. Amiel E, Lovewell RR, O'Toole GA, Hogan DA, Berwin B. Pseudomonas aeruginosa evasion of phagocytosis is mediated by loss of swimming motility and is independent of flagellum expression. Infect Immun. 2010;78(7):2937-45.

9. Bianconi I, Milani A, Cigana C, Paroni M, Levesque RC, Bertoni G, Bragonzi A. Positive signature-tagged mutagenesis in Pseudomonas aeruginosa: tracking patho-adaptive mutations promoting airways chronic infection. PLoS Pathog. 2011;7(2), e1001270.

10. Dubern JF, Cigana C, De Simone M, Lazenby J, Juhas M, Schwager S, Bianconi I, Doring G, Eberl L, Williams P, et al. Integrated whole-genome screening for Pseudomonas aeruginosa virulence genes using multiple disease models reveals that pathogenicity is host specific. Environ Microbiol. 2015:17:4379-4393.

11. Smith EE, Buckley DG, Wu Z, Saenphimmachak C, Hoffman LR, D'Argenio DA Miller SI, Ramsey BW, Speert DP, Moskowitz SM, et al. Genetic adaptation by Pseudomonas aeruginosa to the airways of cystic fibrosis patients. Proc Natl Acad Sci U S A. 2006:103(22):8487-92.

12. Drumm ML, Konstan MW, Schluchter MD, Handler A, Pace R, Zou F, Zariwala M, Fargo D, Xu A, Dunn JM, et al. Genetic modifiers of lung disease in cystic fibrosis. N Engl J Med. 2005;353(14):1443-53.

13. Guillot L, Beucher J, Tabary O, Le Rouzic P, Clement A, Corvol H. Lung disease modifier genes in cystic fibrosis. Int J Biochem Cell Biol. 2014;52:83-93.

14. Cheng SL, Yu CJ, Chen CJ, Yang PC. Genetic polymorphism of epoxide hydrolase and glutathione S-transferase in COPD. Eur Respir J. 2004;23(6):818-24

15. Demeo DL, Campbell EJ, Barker AF, Brantly ML, Eden E, McElvaney NG, Rennard SI, Sandhaus RA, Stocks JM, Stoller JK, et al. IL10 polymorphisms are associated with airflow obstruction in severe alpha1-antitrypsin deficiency. Am J Respir Cell Mol Biol. 2008;38(1):114-20.

16. Gruenheid S, Gros P. Forward genetic dissection of innate response to infection in inbred mouse strains: selected success stories. Clin Exp Immunol. 2010;162(3):393-401.

17. Vidal SM, Malo D, Marquis JF, Gros P. Forward genetic dissection of immunity to infection in the mouse. Annu Rev Immunol. 2008:26:81-132.

18. Pennington JE, Williams RM. Influence of genetic factors on natural resistance of mice to Pseudomonas aeruginosa. J Infect Dis. 1979;139(4):396-400

19. Morissette C, Skamene E, Gervais F. Endobronchial inflammation following Pseudomonas aeruginosa infection in resistant and susceptible strains of mice. Infect Immun. 1995;63(5):1718-24.

20. Morissette C, Francoeur C, Darmond-Zwaig C, Gervais F. Lung phagocyte bactericidal function in strains of mice resistant and susceptible to Pseudomonas aeruginosa. Infect Immun. 1996;64(12):4984-92.

21. Sapru K, Stotland PK, Stevenson MM. Quantitative and qualitative differences in bronchoalveolar inflammatory cells in Pseudomonas aeruginosa-resistant and -susceptible mice. Clin Exp Immunol. 1999;115(1):103-9.

22. Tam M, Snipes GJ, Stevenson MM. Characterization of chronic bronchopulmonary Pseudomonas aeruginosa infection in resistant and susceptible inbred mouse strains. Am J Respir Cell Mol Biol. 1999;20(4):710-9. 
23. De Simone M, Spagnuolo L, Lore NI, Rossi G, Cigana C, De Fino I, Iraqi FA, Bragonzi A. Host genetic background influences the response to the opportunistic Pseudomonas aeruginosa infection altering cell-mediated immunity and bacterial replication. PLoS One. 2014;9(9), e106873.

24. Lore NI, Cigana C, De Fino I, Riva C, Juhas M, Schwager S, Eberl L, Bragonzi A. Cystic fibrosis-niche adaptation of Pseudomonas aeruginosa reduces virulence in multiple infection hosts. PLoS One. 2012;7(4), e35648.

25. Darvasi A, Soller M. Selective DNA pooling for determination of linkage between a molecular marker and a quantitative trait locus. Genetics. 1994;138(4):1365-73.

26. Iraqi F. Fine mapping of quantitative trait loci using advanced intercross lines of mice and positional cloning of the corresponding genes. Exp Lung Res. 2000;26(8):641-9.

27. Iraqi FA, Behnke JM, Menge DM, Lowe AM, Teale AJ, Gibson JP, Baker LR, Wakelin DR. Chromosomal regions controlling resistance to gastro-intestinal nematode infections in mice. Mamm Genome. 2003;14(3):184-91.

28. Menge DM, Behnke JM, Lowe A, Gibson JP, Iraqi FA, Baker RL, Wakelin D. Mapping of chromosomal regions influencing immunological responses to gastrointestinal nematode infections in mice. Parasite Immunol. 2003;25(6):341-9.

29. Hernandez-Valladares M, Naessens J, Gibson JP, Musoke AJ, Nagda S, Rihet P, Ole-MoiYoi OK, Iraqi FA. Confirmation and dissection of QTL controlling resistance to malaria in mice. Mamm Genome. 2004;15(5):390-8.

30. Iraqi F, Clapcott SJ, Kumari P, Haley CS, Kemp SJ, Teale AJ. Fine mapping of trypanosomiasis resistance loci in murine advanced intercross lines. Mamm Genome. 2000;11(8):645-8.

31. Iraqi FA, Athamni H, Dorman A, Salymah Y, Tomlinson I, Nashif A, Shusterman A, Weiss E, Houri-Haddad Y, Mott R, et al. Heritability and coefficient of genetic variation analyses of phenotypic traits provide strong basis for high-resolution QTL mapping in the Collaborative Cross mouse genetic reference population. Mamm Genome. 2014;25(3-4):109-19.

32. Haley CS, Knott SA. A simple regression method for mapping quantitative trait loci in line crosses using flanking markers. Heredity (Edinb). 1992;69(4):315-24.

33. Churchill GA, Doerge RW. Empirical threshold values for quantitative trait mapping. Genetics. 1994;138(3):963-71.

34. Dupuis J, Siegmund D. Statistical methods for mapping quantitative trait loci from a dense set of markers. Genetics. 1999;151(1):373-86.

35. Broman KW, Wu H, Sen S, Churchill GA. R/qtl: QTL mapping in experimental crosses. Bioinformatics. 2003:19(7):889-90.

36. Aerts S, Lambrechts D, Maity S, Van Loo P, Coessens B, De Smet F, Tranchevent LC, De Moor B, Marynen P, Hassan B, et al. Gene prioritization through genomic data fusion. Nat Biotechnol. 2006;24(5):537-44.

37. Tranchevent LC, Barriot R, Yu S, Van Vooren S, Van Loo P, Coessens B, De Moor B, Aerts S, Moreau Y, et al. ENDEAVOUR update: a web resource for gene prioritization in multiple species. Nucleic Acids Res. 2008;36(Web Server issue):W377-84.

38. Moreau Y, Tranchevent LC. Computational tools for prioritizing candidate genes: boosting disease gene discovery. Nat Rev Genet. 2012;13(8):523-36.

39. Zhan $Y$, Zhang R, Lv H, Song X, Xu X, Chai L, Lv W, Shang Z, Jiang Y. Prioritization of candidate genes for periodontitis using multiple computational tools. J Periodontol. 2014;85(8):1059-69.

40. Lorè N, Iraqi FA, Bragonzi A. Collaborative Cross mice for dissecting host genetic determinants of Pseudomonas aeruginosa pneumonia severity. BMC Genet. 2015; 16(106). doi: 10.1186/s12863-015-0260-6.

41. Bragonzi A, Wiehlmann L, Klockgether J, Cramer N, Worlitzsch D, Döring G, Tümmler B. Sequence diversity of the mucABD locus in Pseudomonas aeruginosa isolates from patients with cystic fibrosis. Microbiology. 2006;152(Pt 11):3261-9.

42. Bragonzi A, Paroni M, Nonis A, Cramer N, Montanari S, Rejman J, Di Serio C, Döring $\mathrm{G}$ and Tümmler B. Pseudomonas aeruginosa microevolution during cystic fibrosis lung infection establishes clones with adapted virulence. AJRCCM. 2009;180(2):138-45.

43. Lorè N, Cigana C, De Fino I, Riva C, Juhas M, Schwager S, Eberl L, Bragonzi A. Cystic Fibrosis-Niche Adaptation of Pseudomonas aeruginosa Reduces Virulence in Multiple Infection Hosts. PlosOne. 2012;7(4), e35648.

44. Cigana C, Lorè NI, Riva C, De Fino I, Spagnuolo L, Sipione B, Rossi G, Nonis A, Cabrini G, Bragonzi A. Tracking the immunopathological response to Pseudomonas aeruginosa during respiratory infections. Scientific Report. 2016; 6(21465). doi: 10.1038/srep21465
45. Elbers CC, Onland-Moret NC, Franke L, Niehoff AG, van der Schouw YT, Wijmenga C. A strategy to search for common obesity and type 2 diabetes genes. Trends Endocrinol Metab. 2007;18(1):19-26.

46. Tremblay K, Lemire M, Potvin C, Tremblay A, Hunninghake GM, Raby BA, Hudson TJ, Perez-Iratxeta C, Andrade-Navarro MA, Laprise C. Genes to diseases (G2D) computational method to identify asthma candidate genes. PLoS One. 2008;3(8), e2907.

47. Shinohara H, Inoue A, Toyama-Sorimachi N, Nagai Y, Yasuda T, Suzuki H, Horai R, Iwakura Y, Yamamoto T, Karasuyama H. Dok-1 and Dok-2 are negative regulators of lipopolysaccharide-induced signaling. J Exp Med. 2005;201(3):333-9.

48. Worgall S, Heguy A, Luettich K, O'Connor TP, Harvey BG, Quadri LE, Crystal RG. Similarity of gene expression patterns in human alveolar macrophages in response to Pseudomonas aeruginosa and Burkholderia cepacia. Infect Immun. 2005:73(8):5262-8.

49. Mashima R, Arimura S, Kajikawa S, Oda H, Nakae S, Yamanashi Y. Dok adaptors play anti-inflammatory roles in pulmonary homeostasis. Genes Cells. 2013;18(1):56-65.

50. Mashima R, Honda K, Yang Y, Morita Y, Inoue A, Arimura S, Nishina H, Ema $H$, Nakauchi $H$, Seed B, et al. Mice lacking Dok-1, Dok-2, and Dok-3 succumb to aggressive histiocytic sarcoma. Lab Invest. 2010;90(9):1357-64.

51. Williams R, Zou X, Hoyle GW. Tachykinin-1 receptor stimulates proinflammatory gene expression in lung epithelial cells through activation of NF-kappaB via a G(q)-dependent pathway. Am J Physiol Lung Cell Mol Physiol. 2007;292(2):L430-7.

52. DeRose V, Robbins RA, Snider RM, Spurzem JR, Thiele GM, Rennard SI, Rubinstein I. Substance P increases neutrophil adhesion to bronchial epithelial cells. J Immunol. 1994;152(3):1339-46.

53. Munoz M, Covenas R. Involvement of substance P and the NK-1 receptor in human pathology. Amino Acids. 2014;46(7):1727-50.

54. Verdrengh $M$, Tarkowski $A$. The impact of substance $P$ signalling on the development of experimental staphylococcal sepsis and arthritis. Scand J Immunol. 2008;67(3):253-9.

55. Hazlett LD, McClellan SA, Barrett RP, Liu J, Zhang Y, Lighvani S. Spantide I decreases type I cytokines, enhances IL-10, and reduces corneal perforation in susceptible mice after Pseudomonas aeruginosa infection. Invest Ophthalmol Vis Sci. 2007:48(2):797-807.

56. de Jong MA, Vriend LE, Theelen B, Taylor ME, Fluitsma D, Boekhout T, Geijtenbeek TB. C-type lectin Langerin is a beta-glucan receptor on human Langerhans cells that recognizes opportunistic and pathogenic fungi. Mol Immunol. 2010;47(6):1216-25.

57. Collaco JM, Cutting GR. Update on gene modifiers in cystic fibrosis. Curr Opin Pulm Med. 2008;14(6):559-66.

58. Cutting GR. Modifier genes in Mendelian disorders: the example of cystic fibrosis. Ann N Y Acad Sci. 2010;1214:57-69.

59. Corken A, Russell S, Dent J, Post SR, Ware J. Platelet glycoprotein Ib-IX as a regulator of systemic inflammation. Arterioscler Thromb Vasc Biol. 2014;34(5):996-1001.

60. Wu TT, Tai YT, Cherng YG, Chen TG, Lin CJ, Chen TL, Chang HC, Chen RM. GATA-2 transduces LPS-induced il-1beta gene expression in macrophages via a toll-like receptor 4/MD88/MAPK-dependent mechanism. PLoS One. 2013;8(8), e72404.

\section{Submit your next manuscript to BioMed Central and we will help you at every step:}

- We accept pre-submission inquiries

- Our selector tool helps you to find the most relevant journal

- We provide round the clock customer support

- Convenient online submission

- Thorough peer review

- Inclusion in PubMed and all major indexing services

- Maximum visibility for your research

Submit your manuscript at www.biomedcentral.com/submit 